\title{
Förderung der Insulinsekretion in vitro durch ein neues, hochwirksames Antidiabetikum
}

\author{
H. Schwarz, J. Ammon*, J.-E. Yeboah, H.E. Hirdebrandt und E. F. Pfeiffer**
}

Abteilung für Innere Medizin, Endokrinologie und Stoffwechsel des Zentrums für Innere Medizin der Universität Ulm (Medizinisch-Naturwissenschaftliche Hochschule

Eingegangen am 5. April 1967

Stimulation of Insulin Secretion in vitro by a New and Highly Active Hypoglycaemic Agent

Summary. The ability of a new and highly potent sulfonylurea derivative (HB 419) to stimulate insulin secretion in vitro was tested in our laboratory. This derivative, $\mathrm{N}$-[4-(B-<2 methoxy-5-chlorbenzamido $\rangle$-ethyl) -benzolsulfonyl]- $\mathrm{N}^{\prime}$-cyclohexylurea, already shown to be effective in vivo, was tried in vitro on isolated slices of pancreases of rats and rabbits. - It was found that 0.1 $\mu \mathrm{g} / \mathrm{ml}$ of HB 419 produced a significant increase in insulin secretion following its administration. In rabbit pancreas slices in vitro, the presence of $10 \mu \mathrm{g} \mathrm{HB} 419$ resulted in maximum stimulation, while in rat pancreas slices maximum stimulation was not yet reached with $100 \mu \mathrm{g}$ HB $419 \mathrm{ml}$. - In comparison with Tolbutamide, and using insulin secretion in vitro as an index, this derivative appears to be 100 times more effective in rats and 1000 times more effective in rabbits. - These results are in good agreement with the in vivo studies reported by others.

Stimulation de la sécrétion insulinique in vitro par un nouvel agent hypoglycémiant dे haute activité spécifique

Résumé. Un nouveau dérivé (HB 419) des sulfamidés hypoglycémiants, la $N$ - $44-(\beta-<2$ méthoxy-5-chloro-benza mido >-éthyl) -benzosulfonyl] $\mathrm{N}^{\top}$ 'cyclohexyl-urée s'est révélé très actif in vivo. In vitro, il provoque également une stimulation de la sécrétion de l'insuline par des tranches de pancréas isolé de rats et de lapins. - Dans les deux cas, $0,1 \mathrm{\gamma} / \mathrm{ml}$ ont suffi à produire une augmenta- tion significative de la sécrétion insulinique. La stimulation maximale est atteinte à la concentration de $10 \% / \mathrm{ml}$ chez le lapin, elle ne l'est pas encore à la concentration de $100 \mathrm{\gamma} / \mathrm{ml}$ chez le rat. - L'effet du nouveau dérivé, comparé avec celui du tolbutamide, est 100 fois plus grand chez le rat et 1000 fois plus grand chez le lapin. Ces résultats correspondent bien à ceux obtenus in vivo par d'autres auteurs.

Zusammenfassung. Ein neues, in vivo sehr wirksames Sulfonylharnstoff-Derivat, $\mathrm{N}-[4-(\beta-<2$ methoxy-5-chlorbenzamido $>$-aethyl) -benzolsulfonyl] - $\mathrm{N}^{\prime}$-cyclo-hexylharnstoff, führte auch in vitro an isolierten Pankreasschnitten von Ratten und Kaninchen zu einer Stimulierung der Insulinausschüttung. - Eine Konzentration von $0,1 \mathrm{\gamma} / \mathrm{ml}$ hat bei beiden Tierspezies bereits einen signifikanten Anstieg der Insulin-Sekretion zur Folge. Während Kaninchen-Pankreata bereits durch $10 \mathrm{\gamma} / \mathrm{ml}$ maximal stimuliert sind, ist bei Ratten ein Maximalwert bei $100 \mathrm{\gamma} / \mathrm{ml}$ noch nicht erreicht. - Das neue Präparat zeigt, verglichen mit Tolbutamid, bei Ratten eine 100- und bei Kaninchen eine 1000-fach stärkere Wirkung. Diese Ergebnisse zeigen gute Übereinstimmung mit in vivo Untersuchungen anderer Autoren.

Key-words: Insulin secretion "in vitro", Radioimmunoassay of Insulin, Immunologically measurable Insulin (IMI), Oral Antidiabetic Substances, Sulfonylureas-N[4-(B- $\langle 2$ methoxy-5-chlorbenzamido $\rangle$-ethyl)-benzolsulfonyl] -N'-Cyclohexylurea (Hb 419), Tolbutamide

\section{Einleitung}

Seit der Aufklärung des Wirkungsmechanismus der Sulfonylharnstoffe durch Loubatiz̀kes [7] und der Verwendung der Sulfonylharnstoffe für therapeutische Maßnahmen, besteht großes Interesse, neue und aktivere Sulfonylharnstoff-Derivate mit geringeren Nebenwirkungen herzustellen. Bei der klinischen Prüfung dieser Präparate bestimmt man neben der Senkung des Blutzuckers auch die Erhöhung des Insulinspiegels im Blut nach parenteraler Applikation. Nach den Untersuchungen von MELANI et al. [9] ist die Erhöhung des Insulinspiegels bereits eine Minute nach der intravenösen Injektion nachzuweisen. Die in vivo Untersuchungen ermöglichen jedoch nur mit Hilfe komplizierter Techniken eine Aussage über den Wirkungsmechanismus von blutzuckersenkenden Substanzen. Untersuchungen an isolierten Pankreas-

\footnotetext{
* Derzeitige Adresse: Institut für Strahlenheilkunde, Frankfurt am Main.

** Ausgeführt mit Unterstützung der Deutschen Forschungsgemeinschaft, Bad Godesberg.
}

geweben bieten dagegen den Vorteil, da $\beta$ sekundäre Wirkungen weitgehend ausgeschlossen sind (PFEIFFER et al. [12]). So konnten Frerichs et al. [3], Telib et al. [14] und Schwarz [11] an der in vitro Präparation eine direkte Stimulierung der Insulinausschüttung durch Sulfonylharnstoffe nachweisen.

Bei der Suche nach neuen Sulfonylharnstoff-Derivaten wurden von AumüLLER et al. [1] ein Benzamido-alkyl-benzolsulfonyl-harnstoff-Derivat (HB 419) aufgefunden, welches bei verschiedenartigen Versuchstieren und beim Menschen schon in Dosen von weniger als $0.1 \mathrm{mg} / \mathrm{kg}$ Körpergewicht den Blutzucker zu senken vermag. Im Rabmen dieser Untersuchungen sollte nun der Einfluß des neuen, hochwirksamen. Sulfonylharnstoff-Derivates auf die Insulinausschüttung in vitro bestimmt und mit anderen, bereits bekannten Derivaten verglichen werden.

\section{Material und Methodik}

Bei den bisherigen Untersuchungen der Insulinsekretion in vitro mußte mit der insulinzerstörenden 
Wirkung von proteolytischen Fermenten des exokrinen Pankreasparenchyms gerechnet werden. Deshalb gibt es verschiedene Vorschläge zum Schutz des Insulins während der Inkubation. So verwendeten CANDELA et al. [2] Albumin als Zusatz zum Inkubationsmedium, Humber et al. [6] sind der Ansicht, daß nur mit Geweben, welche frei von exokrinem Parenchym sind, gearbeitet werden sollte. Wir haben ein von MaLaIssE et al. [15] vorgeschlagenes Verfahren modifiziert und diesen Versuchsbedingungen angepaßt.

Bei der neuen Technik wird das freigesetzte Insulin vor dem Einfluß der proteolytischen Fermente durch Insulinantikörper geschützt. Nach ausführlichen Untersuchungen von HrLDEBRANDT et al. [5] von unserer Gruppe bleibt der Komplex: Insulinantikörper - Insulin stabil gegenüber der Wirkung von PankreasHomogenaten. Zum Nachweis der ausgeschütteten und an Antikörper gebundenen Insulinmenge eignet sich eine radioimmunologische Technik. Die Trennung des freien vom gebundenen Insulin wurde mit Hilfe von Dextran 80 beladener Aktivkohle durchgeführt (HkRBERT et al. [4]).

\section{a) Herstellung der Antikörper}

Das Anti-Insulin-Serum wurde von Meerschweinchen gewonnen. Die Immunisierung der Tiere erfolgte mit Insulin vom Schwein, kombiniert mit komplettem Freund'schen Adjuvans (Kat. Nr. 70-156, Hyland Lab., Los Angeles).

Den Tioren wurde in wöchentlichen Abständen 4 mal subcutan eine Mischung von 0,5 ml Adjuvans mit $0,5 \mathrm{ml}$ Insulinlösung injiziert. 10-14 Tage nach der letzten Injektion wurden die Tiere durch Herzpunktion entblutet und die Antikörperkonzentration nach einem von HILDEBRANDT et al. [5] entwickelten Verfahren bestimmt.

\section{b) Pufferlösung}

Für die Inkubation und die radioimmunologische Insulinbestimmung wurde ein Puffer folgender Zusammensetzung verwendet:

$0.145 \mathrm{M} / 1 \mathrm{NaCl}$, p.a.

$0.5 \%$ Rinderalbumin, trocken, reinst

$\mathrm{M} / 100 \mathrm{~K}_{2} \mathrm{HPO}_{4}\left(3 \mathrm{H}_{2} \mathrm{O}\right)$, Behringwerke

$1.18 \cdot 10^{-3} \mathrm{M} / 1 \mathrm{NaHCO}_{3}$, p.a.

$\mathrm{pH}$ ca. 7.5

\section{c) Isolierung und Inkubation der Gewebe}

Wir verwendeten Pankreasgewebe von männlichen Bastard-Kaninchen (3 kg) und von männlichen Wistar-Ratten $(150 \mathrm{~g})$. Die Isolierung der Pankreata erfolgte unter größter Schonung des Gewebes. Bei den Kaninchen wurde die Bauchspeicheldrüse in drei Teile (Caput, Corpus, Cauda) zerlegt und von jedem Teil je ein 30-35 mg schweres Gewebestiuckchen in Inkubationsgefäße gebracht. Die Rattenpankreata zerschnitten wir, einer Anregung von SoTTER et al. [13] folgend, in etwa $10 \mathrm{mg}$ schwere Stückchen. In jedes Inkubationsgefäß gelangte ein solches Stückchen, insgesamt 8 Schnitte von 8 verschiedenen Ratten. Durch dieses Vorgehen sollte die inhomogene Inselverteilung im Ratten- und Kaninchenpankreas ausgeglichen werden. Ein Inkubationsgefäß enthielt, wie Kontrollmessungen zeigten, $100 \pm 10 \mathrm{mg}$ Pankreasgewebe. Die Inkubation erfolgte in dem bereits erwähnten Puffer, das Volumen eines Ansatzes betrug $4 \mathrm{ml}$, jeder Ansatz enthielt $1 \lambda$ Antikörper. Die Inkubationszeit dauerte bei den Ratten in der Regel 2 Stunden, bei den Kaninchen 3 Stunden.

\section{d) J131.Insulin}

Das radioaktive Insulin stellte uns freundlicherweise die Firma Hoechst zur Verfügung. Die spezifische Aktivität des markierten Schweine-Insulins betrug $100 \mathrm{mC} / \mathrm{mg}$. Mit dem bereits beschriebenen Puffer wurde das markierte Insulin so verdünnt, daß die Aktivität pro ml etwa $50000 \mathrm{Impulse} / \mathrm{min}$ aufwies. Bei $100 \mathrm{mC} / \mathrm{mg}$ spezifischer Aktivität sind damit in $1 \mathrm{ml}$ ca. $10 \mu \mathrm{E}$ markiertes Insulin enthalten.

\section{e) Aktivkohle}

Zur Herstellung der mit Dextran 80 beladenen Aktivkohle verwendeten wir den bereits erwähnten Puffer, jedoch ohne Albuminzusatz. Wir lösten dabej

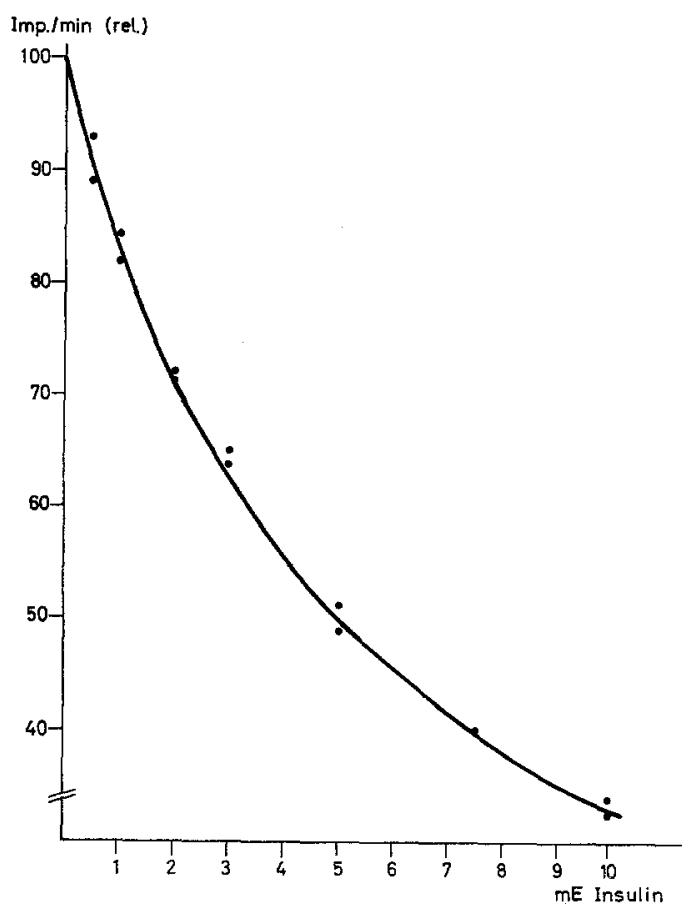

Abb. 1. Radioimmunologische Bestimmung von Insulin: Die Antikörpermenge eines Ansatzes beträgt $1 \lambda$. Damit ist der Meßbereich zwischen 0 und $10 \mathrm{mEE}$ Insulin / Probe festgelegt

$0.5 \mathrm{~g}$ Dextran in $200 \mathrm{ml}$ Puffer und gaben anschließend. $20 \mathrm{~g}$ Aktivkohle hinzu. Diese Suspension war pipettierbar, sie mußte jedoch während des Gebrauches mit einem Magnetrübrer stetig gemischt werden. 
f) Durchführung der Bestimmungen

Zunächst stellten wir Albuminpuffer mit $1 \lambda$ Antikörper/4 ml her. Danach wurden bei jeder Versuchsreihe je $4 \mathrm{ml}$ von dem reinen Puffer, bzw. von den verschiedenen Konzentrationen der Sulfonylharnstoffe (im gleichen Puffer gelöst) in die Inkubationsgläschen

Tabelle 1a. Die Tabelle zeigt die Absolutwerte, welche für Pankreata von Kaninchen bei der Bestimmung der Insulinausschüttung in Abhängigkeit von der Konzentration erhalten wurden

\begin{tabular}{|c|c|c|c|c|c|c|}
\hline & \multicolumn{5}{|c|}{ تВ $419 \gamma / \mathrm{ml}$} & \multirow{2}{*}{$\frac{\begin{array}{l}\text { Tolbuta- } \\
\mathrm{mid} \\
\mathrm{mg} / \mathrm{ml}\end{array}}{1.0}$} \\
\hline $\begin{array}{l}\text { Kon- } \\
\text { zentraw } \\
\text { tion }\end{array}$ & 0 & 0.1 & 1.0 & 10.0 & 100.0 & \\
\hline $\begin{array}{l}\text { Mittel- } \\
\text { wert }\end{array}$ & 3300 & 4680 & 5500 & 5900 & 5380 & 5560 \\
\hline $\begin{array}{l}\text { mittl. } \\
\text { Ab- } \\
\text { weich. }\end{array}$ & \pm 120 & \pm 400 & \pm 460 & \pm 500 & \pm 480 & \pm 340 \\
\hline $\begin{array}{l}\text { Zahl d. } \\
\text { Ver- } \\
\text { suche }\end{array}$ & 47 & 12 & 15 & 16 & 4 & 21 \\
\hline $\begin{array}{l}\text { stat. } \\
\text { Siche- } \\
\text { rung }\end{array}$ & \multicolumn{2}{|c|}{$P<0.02$} & \multicolumn{2}{|c|}{$P>0.05$} & & \\
\hline
\end{tabular}

Zur Aufstellung einer Eichkurve (vgl. Abb. 1) wurden in Wassermannröhrchen, welche $2 \mathrm{ml}$ Puffer mit Antikörpern und $1 \mathrm{ml} \mathrm{J}^{131}$-Insulinlösung enthielten, $1 \mathrm{ml}$ Insulinlösung mit steigender Konzentration pipettiert. Anschließend erfolgte eine weitere Inkubation der Wassermannröhrchen für 2 Stunden bei $37^{\circ} \mathrm{C}$.

Tabelle 1 b. Die Tabelle zeigt die Absolutwerte, welche für Pankreata von Ratten bei der Bestimmung der Insulinausschüttung in Abhängigkeit von der Konzentration er. halten wurden

\begin{tabular}{l|lllll|l}
\hline & HB $419 \gamma / \mathrm{ml}$ & & & & $\begin{array}{l}\text { Tolbuta- } \\
\text { mid } \\
\mathrm{mg} / \mathrm{ml}\end{array}$ \\
\hline $\begin{array}{l}\text { Kon- } \\
\text { zentra- } \\
\text { tion }\end{array}$ & 0 & 0.1 & 1.0 & 10.0 & 100.0 & 1.0 \\
$\begin{array}{l}\text { Mittel- } \\
\text { wert } \\
\text { mittl. } \\
\text { Ab- } \\
\text { weich. }\end{array}$ & 2280 & 2960 & 3140 & 3720 & 4360 & 4020 \\
$\begin{array}{l}\text { Zahl d. } \\
\begin{array}{l}\text { Ver- } \\
\text { suche }\end{array}\end{array}$ & 45 & 14 & 7 & 13 & 11 & 16 \\
$\begin{array}{l}\text { stat. } \\
\begin{array}{l}\text { Siche- } \\
\text { rung }\end{array}\end{array}$ & $P<0.02$ & $P<0.02$ & \pm 160 & \pm 200 & \pm 180 & \pm 300 \\
\hline
\end{tabular}

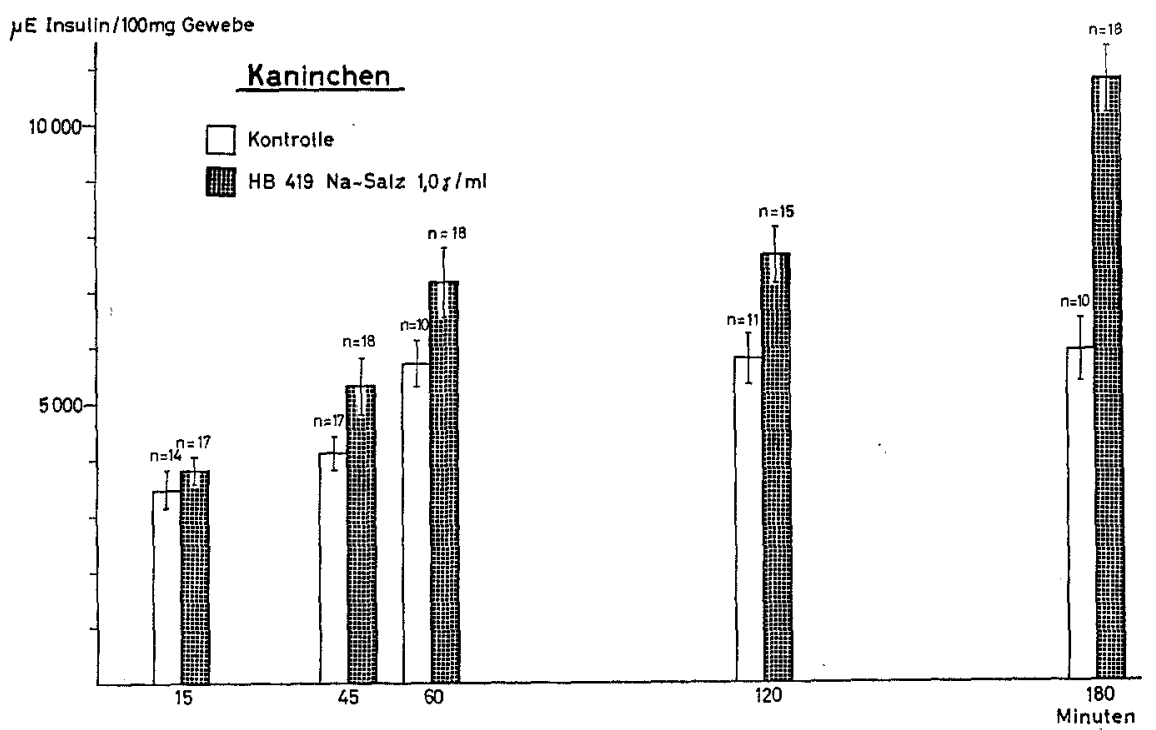

Abb. 2a. Insulinsekretion in Abhängigkeit von der Inkubationszeit: Bei Pankreata von Kaninchen ist nach 3 Stunden ein optimaler Unterschied zwischen dem In-sulingehalt des Inkubationsmediums ohne und mit $1.0 \mathrm{\gamma} / \mathrm{ml} \mathrm{HB} 419$ als Na-Salz nachzuweisen

pipettiert. Die Inkubationsgefäße $(25 \mathrm{ml})$ wurden nach dem Einbringen der Gewebe in einemWarburgGerät bis zu 3 Stunden bei $37^{\circ} \mathrm{C}$ inkubiert.

Nach der Inkubation wurden von jedem Ansatz $2 \mathrm{ml}$ Inkubationsmedium in Wassermannröhrchen pipettiert, welohe bereits $1 \mathrm{ml}$ der J131-Insulinlösung und $1 \mathrm{ml}$ Albuminpuffer ohne Antikörper enthielten.
Nach Hinzufügen von $2 \mathrm{ml}$ der Aktivkohle-Suspension in jedes Röhrchen, kurzem Aufschütteln und Zentrifugieren verwendeten wir $2 \mathrm{ml}$ Überstand zur Alktivitätsbestimmung.

Mit den Insulin-Standardlösungen konnte die in Abb. 1 dargestellte Eichkurve erhalten werden. Die für die einzelnen Ansätze aus der Kurve abgelesenen 
Insulinwerte mußten verdoppelt werden, da von den $4 \mathrm{ml}$ Inkubationsmedium nur $2 \mathrm{ml}$ zur radioaktiven Bestimmung Verwendung fanden.

\section{g) Auswertung der Ergebnisse}

Bei der Durchführung der Untersuchungen war es erforderlich, jede Versuchsreihe mehrfach zu wiederholen. Zur graphischen Darstellung und statistischen

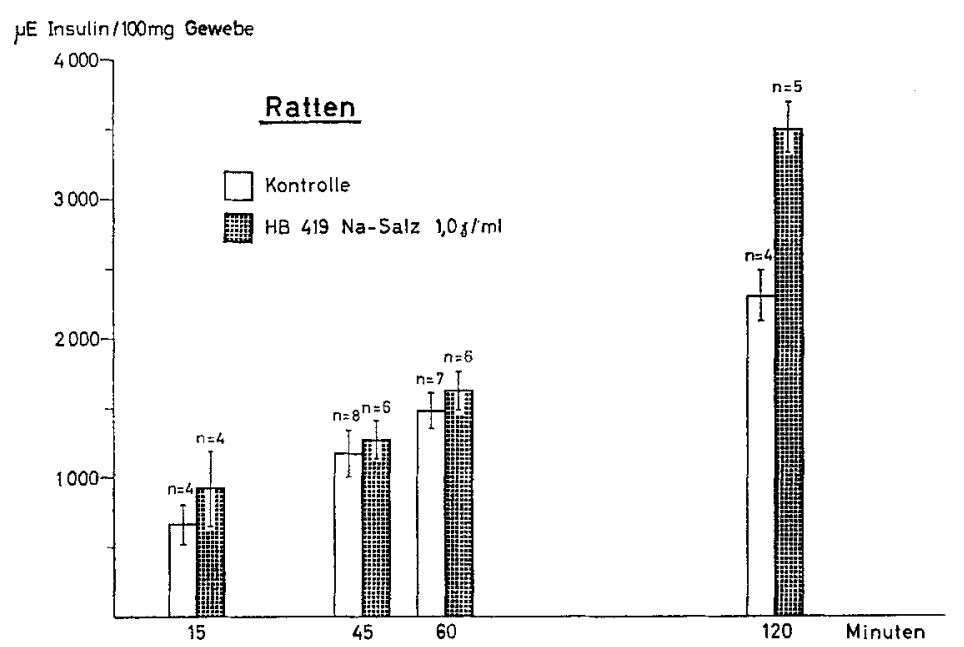

Abb, $2 \mathrm{~b}$. Insulinsekretion in Abhängigkeit von der Inkubationszeit: Bei Pankreata von Ratten ist nach 2 Stunden ein optimaler Unterschied zwischen dem Insulingehalt des Inkubationsmediums ohne und mit $1.0 \gamma / \mathrm{ml} \mathrm{HB} 419$ als Na-Salz nachzuweisen

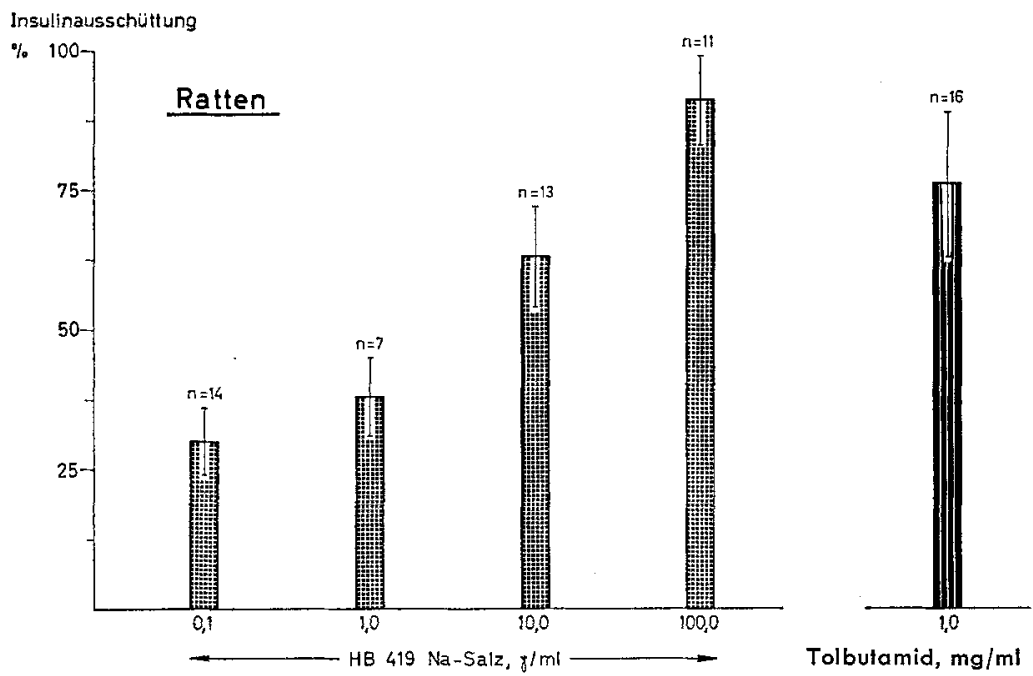

Abb. 3a. Insulinausschüttung in Abhängigkeit von der Konzentration: Die Stimulierung der Insulinausschüttung von Kaninchen-Pankreata wird bereits bei $0.1 \gamma / \mathrm{ml}$ HB 419 signifikant gegenüber dem Null-Wert $(P<0.02)$. Die Unterschiede der Ergebnisse zwischen 1.0, 10.0 oder $100 \mathrm{\gamma} / \mathrm{ml}$ sind rein zufällig $(P>0.05)$

Auswertung der in Tab. 1 dargestellten Ergebnisse wurde die Insulinausschüttung der Gewebe in reinem Puffermedium gleich $100 \%$ gesetzt. Die Differenz zwischen dem Leerwert und der Insulinmenge in dem Medium mit der zu untersuchenden Substanz ist bei den Abbildungen auf der Ordinate als „Insulinausschüttung" in Prozenten dargestellt.

\section{Ergebnisse}

Bei den Versuchen sollten die Konzentrationen der einzelnen Substanzen so gewählt werden, daß es möglich war, einen Schwellen- und Maximalwert zu erfassen. In einigen Fällen war ein Maximalwert nicht zu ermitteln, da nicht mehr von der betreffenden Substanz in Lösung ging. Weiterhin wurde bei einer mittleren Konzentration die optimale Inkubationszeit festgestellt.

Die in Abb. 1 gezeigte Eichkurve läßt einen Meßbereich zwischen 0 und $10000 \mu \mathrm{E} z u$. Damit war es möglich, sämtliche Stimulationseffekte zu erfassen.

a) Ermittlung der optimalen Inkubationszeit

Die Ergebnisse der optimalen Inkubationszeit für Ratten und Kaninchen sind in den Abb. 2 a und $2 \mathrm{~b}$ zusammengestellt. In beiden $\mathrm{Ab}$ bildungen ist auf den Ordinaten der Insulingehalt des Inkubationsmediums eingetragen. Aus dem Vergleich des Leerwertes mit demjenigen von $1.0 \gamma / \mathrm{ml}$ HB 419 geht hervor, daß bei Kaninchenpankreas der beste Stimulationseffekt nach 3 Stunden eintritt. Ein signifikanter Anstieg war bereits nach 45 min nachzuweisen.

Bei Ratten betrug, wie aus $\mathrm{Abb}$. $2 \mathrm{~b}$ hervorgeht, die optimale Inkubationszeit $120 \mathrm{~min}$. Diese Zeiten stimamen auch mit den Frgebnissen anderer Autoren überein (Mrache [10], Frerichs [3]).

b) Bestimmung der Stimulationswirkung von $H B$ 419.

Die Stimulationswirkung verschiedener Konzentrationen von $\mathrm{HB}$ 419 Na-Salz wurde für Kaninchen in $\mathrm{Abb} .3 \mathrm{a}$ und Tab. 1 a dargestellt. Die Schwellenkonzentration lag bei $0.1 \gamma / \mathrm{ml}$, eine Konzentration von $0.01 \gamma / \mathrm{ml}$ zeigte keinen gegenüber dem Leerwert signifikanten Fffekt. Der Maximalwert wurde bereits bei $10.0 \gamma / \mathrm{ml}$ erreicht, höhere Konzentrationen zeigten keine stärkere Wirkung. Beim Vergleich mit Tolbutamid - Konzentration

\footnotetext{
I Freundlicherweise zur Verfügung gestellt von Dr. RolaAND MÜLLER, Farbwerke Hoechst.
} 
$1 \mathrm{mg} / \mathrm{ml}$ - zeigte sich eine Wirkung, die mit derjenigen von $1.0 \gamma / \mathrm{ml} \mathrm{HB} 419$ vergleichbar ist.

Für Ratten wurden die in Abb. 3 b und Tab. $1 \mathrm{~b}$ dargestellten Ergebnisse erhalten. Der Schwellenwert lag bei diesen Versuchen ebenfalls bei $0.1 \gamma / \mathrm{ml}$, der Maximalwert betrug jedoch $100 \gamma / \mathrm{ml}$. Es ist möglich, daß bei einer stärkeren Konzentration der Wert noch höher liegt, es konnte jedoch in dem verwendeten Puffer nicht mehr HB 419 gelöst werden. Bei den Ratten bewirkten $10.0 \gamma / \mathrm{ml}$ ungefähr denselben Stimulationseffekt wie $1.0 \mathrm{mg} / \mathrm{ml}$ Tolbutamid.

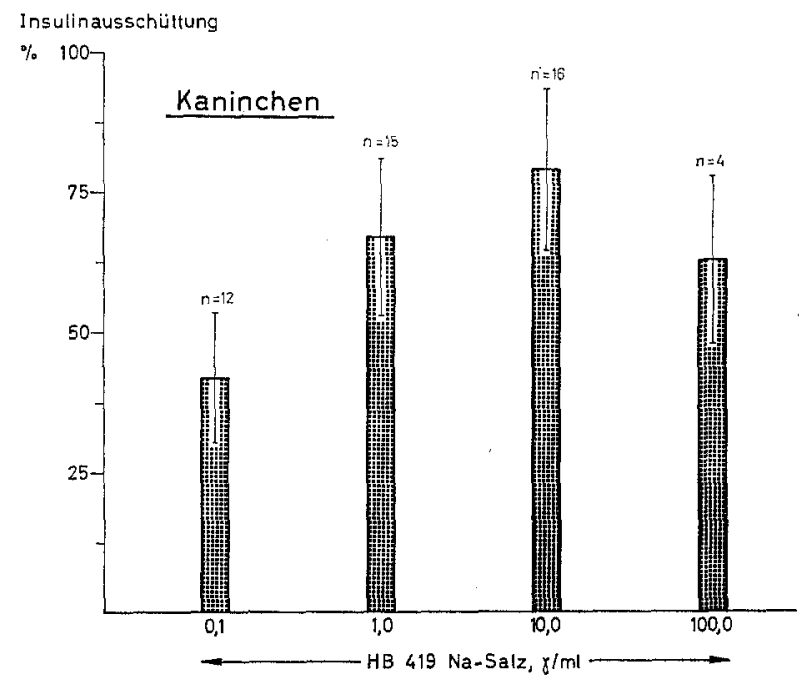

Abb. 3 b. Insulinausschüttung in Abhängigkeit von der Konzentration: Die Stimulierung der Insulinausschüttung von Ratten-Pakreata wird bei 0.1 $\gamma / \mathrm{ml} \mathrm{HB} 419 \mathrm{Na-Salz}$ signifikant

\section{Besprechung der Ergebnisse}

Mit Hilfe der beschriebenen, neuen Technik konnte der direkte, stimulierende Effekt von $\mathrm{HB} 419$ als NaSalz nachgewiesen werden. Die vom Gewebe ausgeschütteten Insulinmengen lagen zwischen 4000 und $100000 \mu \mathrm{E} / 100 \mathrm{mg}$ Gewebe bei Kaninchen und zwischen 1000 und $4000 \mu \mathrm{E} / 100 \mathrm{mg}$ Gewebe bei Ratten.

MeIER et al. [8] von unserer Gruppe konnten im Kaninchenpankreas einen Insulingehalt von $400 \mathrm{mE} / 0.1$ $\mathrm{g}$ nachweisen, im Rattenpankreas $100 \mathrm{mE} / 0.1 \mathrm{~g}$. Aus dem Vergleich der im Gewebe enthaltenen Insulinmenge und der ausgeschütteten Menge geht hervor, daß bei beiden Tierspezies $1-2.5 \%$ des im Gewebe enthaltenen Insulins freigesetzt werden. Bei den bisherigen Verfahren (vgl. PfenfFer et al. [12]) wurden nur $0.1-0.2 \%$ gefunden. Der Unterschied ist in diesem Fall darauf zurückzuführen, daß freigesetztes Insulin durch proteolytische Fermente zerstört werden kann.

Weiterhin kann, wie Untersuchungen von HrLdEBRANDT et al. [5] aus unserem Laboratorium zeigten, das dem Massenwirkungsgesetz unterliegende Gleichgewicht zwischen dem in Ribosomen gespeicherten Insulin und Insulin im Medium verschoben werden. Wird nun das Insulin im Medium durch Antikörper fixiert, so muß
Insulin nachgeliefert werden, hieraus resultieren die höheren Insulinwerte.

Wie aus den Abb. 2 und 3 hervorging, lagen die untersuchten Konzentrationen von HB 419 zwischen 0.1 und $100 \mathrm{\gamma} / \mathrm{ml}$. Um einen unspezifischen Stimulationseffekt, wie z.B. Erhöhung des osmotischen Druckes auszuschalten, wurde Mannit in Konzentrationen bis zu $10 \mathrm{mg} / \mathrm{ml}$ dem Inkubationsmedium zugesetzt. Dabei war kein Stimulationseffekt nachweisbar.

Die mit HB 419 und Rastinon bei der in vitro Prä. paration erhaltenen Ergebnisse wurden mit denjeigenn verglichen, die AUMÜLLER et a1. [1] bei in vivo Versuchen erhielten. In Tab. 2 sind die von AUMứLLER et al. mitgeteilten und die von uns erhaltenen Schwellenwerte eingetragen. Man erkennt, daß sowohl von AUmüLLER als auch von uns bei Ratten eine gegenüber Rastinon um den Faktor 100 stärkere Wirkung von HB 419 festzustellen war (vgl. Abb. 3b). Bei Kaninchen lag der Faktor bei 1000 , dies geht auch aus Abb. 3a hervor. In diesem Zusam. menhang sei erwähnt, daß FrERIcHs et al. [21] am isolierten Pankreas der Ratte ebenfalls durch Tolbutamid einen geringeren Stimulationseffekt als bei Kaninchen beobachteten.

Durch die vorliegenden Untersuchungen konnte gezeigt werden, daß der neue Sulfonylharnstoff HB 419 Na-Salz, der, wie AUMÜLLER zeigte, gegenüber Tolbutamid eine geringere Toxizität aufweist, auch bei der in vitro Präparation die annähernd 1000-fach stärkere Wirkung besitzt.

Tabelle 2. Vergleich der bei Ratten und Kaninchen in vivo (perorale Applikation) und in vitro erforderlichen Mindestmengen von HB 419, bzw. Tolbutamid zum Nachweis einer Wirkung

\begin{tabular}{l|ll|ll}
\hline & \multicolumn{3}{|l|}{ Hb $419 \mathrm{Na}$-Salz } & Tolbutamid \\
\cline { 2 - 4 } p. os & in vitro & p. os & in vitro \\
\hline $\begin{array}{l}\text { Kanin- } \\
\text { chen }\end{array}$ & $25 \gamma / \mathrm{kg}$ & $0.1 \gamma / \mathrm{ml}$ & $25 \mathrm{mg} / \mathrm{kg}$ & $0.1 \mathrm{mg} / \mathrm{ml}$ \\
Ratten & $100 \gamma / \mathrm{kg}$ & $0.1 \gamma / \mathrm{ml}$ & $6.25 \mathrm{mg} / \mathrm{kg}$ & $0.01 \mathrm{mg} / \mathrm{ml}$ \\
\hline
\end{tabular}

\section{Literatur}

1. Aumüller, A., A. Bänder, W. Pfaff, F.H. SCHMIDT, H. WEBER und R. WEYER: Ein neues hochwirksames orales Antidiabeticum. Arzneimittelforsch. 16, 1640-1641 (1966)

2. Candela, J.L.R., und H.G. Coore: Insulinsecretion in vitro. In: Handbuch des Diabetes mellitus, Pathophysiologie und Klinik. Band 1. Hrsg. E.F. PFerfiter, München, Lehmanns Verlag, 1967 (im Druck).

3. Frerichs, H., U. Reich und W. Creutzfeld: Insulinsekretion in vitro. I. Hemmung der glukosein- 
duzierten Insulinabgabe durch Insulin. Klin. Wschr. 43, 136-140 (1965).

4. Herbert, V., Kam-Sexg Lau, Ch. W. Gotmuteb und SH.H. BLEICHER: Coated Charcoal Immunoassay of Insulin. J. Endocr. 25, 1375-1384 (1965).

5. Hildebrandt, H.E., J. Ammon und E.F. Pfeiffer: Quantitative Untersuchungen über die Wirkung von proteolytischen Fermenten der Bauchspeicheldrüse auf Insulin und auf den Komplex Insulin-Insulin. antikörper (in Vorb.).

6. Humbel, R. E.: Proc. nat. Acad. Sci. (USA) 53, $953(1965)$

7. Loubatreres, A.: Analyse du mécanisme de l'action hypoglycémiante du p-aminobenzène-sulfamido-isopropyl-thiodiazol. C. R. Soc. Biol. (Paris) 138, 766 (1944).

8. Mrier, J.M., J. Aymon, U. GRösCheL-STEwart, F. Melant, J.-E. Yeboah und E.F. Pfeiffere: Eine einfache, schnelle Methode zur Bestimmung von Insulin in kleinsten Gewebsmengen (bis $10 \mathrm{mg}$ ). 13 . Symp. Dtsch. Ges. Endocrin., Würzburg 2.-4. 3. 1967, Abstr. Nr. 57 (im Druck).

9. Melani, F., J. Eawecki, K.M. Bartelt und E.F. PFEIFFER: Immunologisch meßbares Insulin (IMT) bei Stoffwechselgesunden, Fettsüchtigen und adipösen Diabetikern nach intravenöser Gabe von Glu. kose, Tolbutamid und Glukagon. Diabetologia 3, 422426 (1967).

10. Mralhe, M.P., et V. Meyer, présentée par M.R. CourRIER: Sécrétion d'insuline par le pancréas du rat in vitro. C. R. Acad. Sci. 253, 1861-1863 (1961).

11. Scrwarz, H.: Untersuchungen über den Einfluß von Glukose und verschiedener Sulfonyl-Harnstoff-Deri- vate auf die Insulinabgabe isolierter Pankreasschnitte von Ratten und Kaninchen (Diss. in Vorb.).

12. Pfeiffer, E.F., M. Telib, J. Ammon, F. Melani und H. Ditschuneit: Direkte Stimulierung der Insulin-Sekretion in vitro durch Sekretin. Dtsch. med. Wschr. 90 1663-1669 (1965).

13. Sutrer, B.: Pers. Mitt.

14. Telis, M., F. Melant, H. Ditschuneit, J. Ammon und E.F. Pfetrater: Vergleichende Untersuchung über die Beeinflussung dor Insulinsekretion isolierter Pankreasgewebe durch Glukose, Sulfonylharnstoffe, ACTH, STH, Glukagon und Sekretin. 12. Symp. Dtsch. Ges. Endocrin., Wiesbaden. 1966

15. Wright, P.H. and W.Y. Malatsse: A Simple Method for the Assay of Guinea Pig Anti-Insulin-Serum. Diabetologia 2, 178-188 (1966).

Prof. Dr. E. F. PhentFfer

Abt. für Innere Medizin, Endokrinologie und Stoffwechsel des Zentrums für Innere Medizin der Universität Ulm 79 Ulm, Donau Safranberg - Steinhövelstr. 9

Sonderdruckanforderungen erbeten an:

Fräulein SerTz

Bibliothek, Zentrum für Innere Medizin, Universität Ulm

79 Ulm (Donau)

Steinhövelstr. 9 